\title{
Relations between two-dimensional models from dimensional reduction
}

\author{
R. L. P. G. Amaral \\ Instituto de Física, Universidade Federal Fluminense, Av. Litoranea, S/N, CEP 24210-340, Niteroi, RJ, Brazil \\ C. P. Natividade \\ Instituto de Física, Universidade Federal Fluminense, Av. Litoranea, S/N, CEP 24210-340, Niteroi, RJ, Brazil \\ Depto de Física e Química, Universidade Estadual Paulista, Campus de Guarantinguetá, CEP 12500-001, Guaratinguetá, SP, Brazil
}

(Received 1 June 1998; published 17 November 1998)

\begin{abstract}
In this work we explore the consequences of dimensional reduction of the 3D Maxwell-Chern-Simons and some related models. A connection between topological mass generation in $3 \mathrm{D}$ and mass generation according to the Schwinger mechanism in 2D is obtained. In addition, a series of relationships is established by resorting to dimensional reduction and duality interpolating transformations. Non-Abelian generalizations are also pointed out. [S0556-2821(98)02022-0]

PACS number(s): 11.15.Tk
\end{abstract}

The search for mechanisms of mass generation to gauge particles without destroying the related symmetry has been one of the most important tasks of theoretical physics in the last decades. Some ingenious strategies have been developed which lead to alternatives to the traditional approach of symmetry breaking. For instance, in three-dimensional spacetimes the addition of a Chern-Simons term to the Maxwell term in the Lagrangian leads to the massive character of the gauge particles [1]. The non-Abelian counterpart of this mechanism is also understood to lead to mass generation although the analysis can be made in terms only of an approximate approach such as $1 / N$ expansion [2].

Generalizations of this mechanism to higher dimensions require the introduction of higher rank gauge fields, indicating that mass generation mechanisms are very sensitive to the underlying dimensionality of spacetime. On the other hand, since long ago the mechanism of Schwinger is known to lead to the massive character of two-dimensional QED [3]. Here the interaction between fermions and gauge fields is the sole mechanism responsible for the phenomenon.

We show here that these mechanisms are in fact closely related. Indeed by a simple dimensional reduction the Maxwell-Chern-Simons Lagrangian is transformed at the bosonized Schwinger model Lagrangian [3]. This illustrates how seemingly distinct phenomena are indeed manifestations in different dimensions of the same basic mechanism. At the same time interesting relations between threedimensional models have been brought about by an interpolating field mechanism. The Maxwell-Chern-Simons (MCS) model, for instance, has been related to the self-dual model $[4,5]$. We explore this relation in this article. The duality transformations are used in $D=3$ and the resulting models are dimensionally reduced. This makes clear some unexpected links between two-dimensional models.

The three-dimensional models with the Chern-Simons action have been useful in the description of the statistical mechanics of planar systems, such as the quantum Hall effect and possibly supercondutivity [6]. The dimensional reduction here discussed may be useful for the description of equilibrium properties of these systems provided the reduction is performed on the time variable leading to the Euclidean versions of the two-dimensional models here dealt with.

Consider three-dimensional Minkowskian spacetime as $\Sigma \times R^{1}$ where $\Sigma$ is a two-dimensional Minkovsky space with arbitrary topology. In this spacetime we take the MaxwellChern-Simons action

$$
L=-\frac{1}{4} F_{\mu \nu} F^{\mu \nu}+\frac{\theta}{2} \epsilon^{\mu \nu \alpha} A_{\mu} F_{\nu \alpha} .
$$

This Abelian vector field is massive $\left(\theta^{2}\right)$ and presents gauge symmetry $\left(A_{\mu} \rightarrow A_{\mu}+(1 / e) \partial_{\mu} \alpha\right)[1]$. This model is a paradigm for so-called topological mass generation where the term "topological" stems from the fact that the second term does not couple to the metric but only to torsion, not contributing to the energy-momentum tensor. Now reduce the spacetime dimension by considering field configurations independent of the variable $x^{2}$ along $R^{1}$ and averaging over it. In this new Lagrangian there is an induced gauge symmetry. Let the " 0 " and " 1 " components be labeled by Latin indices such as " $i, j, k, \ldots$, , and define $\phi=A^{2}$. The $A^{i}$ field remains a vector gauge field while $\phi$ turns out to be a pseudoscalar gauge-invariant field in $\Sigma$. The dynamics of this subspace of solutions of the Lagrangian $L$ is obtained noting that $L$ is reduced to $L^{\prime}$ :

$$
L^{\prime}=-\frac{1}{4} F^{i j} F_{i j}+\frac{1}{2} \partial^{i} \phi \partial_{i} \phi-\theta \phi \epsilon^{i j} F_{i j}
$$

The resulting model [3] is the bosonized version of twodimensional QED as obtained through path integral methods [7]. The $\phi$ field is then interpreted as the scalar associated with the fermion field after its bosonization and the parameter $\theta$ is identified with the charge $e / 2 \sqrt{\pi}$. Its kinetic term comes from the bosonization of the fermion Lagrangian while the interaction term, after integrating by parts, expresses the minimal coupling with the bosonized current $(1 / \sqrt{\pi}) \epsilon^{i j} \partial_{j} \phi$. In this version the mass generation is due to interaction between the fermion and vector fields. These two diverse mechanisms are thus related by dimensional reduction. 
We consider next the addition of the fermionic field in three dimensions:

$$
L=-\frac{1}{4} F_{\mu \nu} F^{\mu \nu}+\frac{\theta}{2} \epsilon^{\mu \nu \alpha} A_{\mu} F_{\nu \alpha}+i \bar{\psi} \not D \psi
$$

After dimensional reduction the Lagrangian turns out to be

$$
L^{\prime}=-\frac{1}{4} F^{i j} F_{i j}+\frac{1}{2} \partial^{i} \phi \partial_{i} \phi-\theta \phi \epsilon^{i j} F^{i j}+i \bar{\psi} \not D \psi-e \bar{\psi} \gamma^{5} \psi \phi
$$

The last term that couples the fermionic to the scalar field in two dimensions is a requirement of dimensional reduction. The fermion field can be bosonized in terms of the pseudoscalar field $\phi^{\prime}$, resulting in the Lagrangian

$$
\begin{aligned}
L^{\prime}= & \frac{-1}{4} F^{i j} F_{i j}+\frac{1}{2} \partial^{i} \phi \partial_{i} \phi-\theta \phi \epsilon^{i j} F_{i j} \\
& +\frac{1}{2} \partial^{i} \phi^{\prime} \partial_{i} \phi^{\prime}+e \mu \phi \sin 2 \sqrt{\pi} \phi^{\prime}
\end{aligned}
$$

where $\mu$ is a regulator parameter.

Dimensional reduction leads to relations between interesting two- and three-dimensional models. At the same time there are some relations between three-dimensional models that have been brought about by the technique of interpolating duality transformations. For instance [4], an interpolating mechanism leads from the (Abelian) Maxwell-Chern-Simons to the self-dual action, which in Minkowski spacetime reads

$$
S_{S D}=\frac{1}{2} a^{\mu} a_{\mu}-\frac{1}{4 \theta} \epsilon_{\mu \nu \alpha} a^{\mu} \partial^{\nu} a^{\alpha},
$$

while an alternative derivation based on the Hamiltonian formalism leads to the same relation [8]. Let us investigate the relationships that can be uncovered from the use of dimensional reduction together with the duality transformation. The direct dimensional reduction of the self-dual action, setting $a^{2}=\varphi$, leads to

$$
L=\frac{1}{2} a^{i} a_{i}-\frac{1}{2} \varphi^{2}+\frac{1}{4 \theta} \epsilon_{i j} f^{i j} \varphi
$$

where $f^{i j}=\partial^{i} a^{j}-\partial^{j} a^{i}$. The integration over the $\varphi$ field gives rise to the Maxwell term for the $a^{i}$ field, resulting in the $D$ $=2$ Proca model. We have thus established a relationship between the bosonized Schwinger model (2) and the Proca model since both are derived through dimensional reduction from models related by duality transformations. The mass spectra of both models are the same. This relationship can be established directly in two-dimensional models, along the lines of [4], as we show now. In the Schwinger model the reexpression of both the Maxwell and the Klein-Gordon terms in terms of auxiliary fields $\varphi$ and $a^{i}$, respectively, is made by replacing the bosonized Schwinger model Lagrangian with the interpolating Lagrangian

$$
L=\frac{1}{2} a^{i} a_{i}-\frac{1}{2} \varphi^{2}-\epsilon_{i j}\left(\varphi \partial^{i} A^{j}-\frac{1}{2} \phi \partial^{i} a^{j}-\theta \phi F^{i j}\right) .
$$

If a translation is made on the auxiliary fields $a^{i} \rightarrow a^{i}$ $+\epsilon^{i j} \partial_{j} \phi$, and $\varphi \rightarrow \varphi-\epsilon^{i j} \partial_{i} A_{j}$, these fields decouple and the bosonized Schwinger model Lagrangian describes the dynamics of the fields $A^{i}$ and $\phi$. With the interpolating Lagrangian it is thus possible to describe the bosonized Schwinger model. On the other hand, the translations on the original fields

$$
\begin{gathered}
A^{i} \rightarrow A^{i}+\frac{1}{2 \theta} a^{i}, \\
\phi \rightarrow \phi+\frac{1}{2 \theta} \varphi,
\end{gathered}
$$

in the interpolating Lagrangian, result in the decoupled Lagrangian

$$
L=\frac{1}{2} a^{i} a_{i}-\frac{1}{2} \varphi^{2}-\epsilon^{i j}\left(\frac{1}{4 \theta} \varphi f_{i j}-\theta \phi F_{i j}\right)
$$

which incorporates the Proca action. The decoupling transformation (9) shows how the fermion field should be treated after bosonization in the dual formulation. For instance the expressions of the fermion field correlation functions in terms of the Mandelstan-like formulas remain the same as in the Schwinger model but with the translation made according to Eqs. (9).

The interpolating mechanism in three dimensions can be extended and should not be restricted to the transformation of the Maxwell term. Let us consider this possibility in some detail. Start with the interpolating (Minkowskian) generating functional

$$
\begin{aligned}
Z[J]= & \int D A^{\mu} D a^{\mu} \exp \left[i \int d ^ { 3 } x \left(-\frac{1}{4}\left(F_{\mu \nu}\right)^{2}\right.\right. \\
& \left.\left.-\frac{\theta}{4} \gamma a_{\mu} S^{\mu \nu} \gamma a_{\nu}+\theta a_{\mu} S^{\mu \nu} \gamma A_{\nu}+J^{\mu} A_{\mu}\right)\right],
\end{aligned}
$$

with $S^{\mu \nu}=\epsilon^{\mu \alpha \nu} \partial_{\alpha}$ and $\gamma$ an arbitrary operator ${ }^{1}$ that commutes with $S^{\mu \nu}$. Making the translation in the $a^{\mu}$ field $a^{\prime} \stackrel{\mu}{=} a^{\mu}+2 \gamma^{-1} A^{\mu}$ it is readily seen that the $a^{\mu}$ field decouples and what remains is the generating functional of the MCS model. If the translation is made however, on the $A^{\mu}$ field, $A^{\mu}=A^{\prime \mu}-\theta(\gamma / \square) S^{\mu \nu} a_{\nu}$, it results in a new factorization of the generating functional:

\footnotetext{
${ }^{1}$ The operator $\gamma$ should satisfy $\int d^{2} x B \gamma A=\int d^{2} x A \gamma B$, for any fields $A$ and $B$. For instance, $\gamma$ can be any power of the D'Alembertian operator.
} 


$$
\begin{aligned}
Z[J]= & \int D A^{\prime \mu} \exp \left[i \int d^{2} x\left(-\frac{1}{4}\left(F_{\mu \nu}^{\prime}\right)^{2}+J^{\mu} A_{\mu}^{\prime}\right)\right] \\
& \times \int D a^{\mu} \exp \left[i \int d ^ { 2 } x \left(-\frac{\theta^{2}}{4} \gamma f_{\mu \nu}(\square)^{-1} \gamma f^{\mu \nu}\right.\right. \\
& \left.\left.-\frac{\theta}{4} \gamma a_{\mu} S^{\mu \nu} \gamma a_{\nu}-\theta J^{\mu} \frac{\gamma}{\square} a_{\mu}\right)\right] .
\end{aligned}
$$

Apart from the contribution of the $A^{\prime}$ Maxwell field the most interesting aspect of the action is encoded in the one for the $a^{\mu}$ field. Contrary to what happens in the MaxwellChern-Simons original action now the highest derivative is associated to the odd-parity operator $S^{\mu \nu}$ and not to the Maxwell like term for $a^{\mu}$. As we have seen the operator $\gamma$ is quite general and can be chosen according to convenience establishing the connection of the MCS model to a family of models.

We apply the dimensional reduction in this dual model. Defining $\varphi=a^{2}$ the resulting action for $a^{\mu}$ reduces to

$$
\begin{aligned}
L= & -\frac{\theta^{2}}{4} \gamma f_{i j}(\square)^{-1} \gamma f^{i j}+\frac{\theta^{2}}{2} \gamma \partial^{i} \varphi(\square)^{-1} \gamma \partial_{i} \varphi \\
& +\frac{\theta}{4} \gamma \varphi \epsilon^{i j} \gamma f_{i j} .
\end{aligned}
$$

Relationships are so established between the (bosonized) Schwinger model and a family of two-dimensional models.

The self-dual action can also be interpreted as the hypermassive limit of the 3D Thirring model. Indeed, it has been recently shown [9] that the partition function for the 3D massive Thirring model is reduced in the one-loop approximation to the self-dual model action. One starts with the Euclidean partition function for the massive Thirring model:

$$
\begin{aligned}
Z_{T h}= & \int D A^{\mu} D \bar{\psi} D \psi \\
& \times \exp \left[-\int d^{3} x\left(\bar{\psi}(i \not \partial+g A+m) \psi+\frac{1}{2 g^{2}}\left(A^{\mu}\right)^{2}\right)\right] .
\end{aligned}
$$

Integration over the fermionic field leads to the fermionic determinant

$$
\ln \left[\operatorname{Det}\left(\frac{i \not D}{i \not \partial}\right)\right]=\sum_{n=1}^{n=\infty} \frac{(-1)^{n+1}}{n} \operatorname{tr}\left(\frac{A}{i \not \partial+m}\right)^{n} .
$$

At the one-loop level the computation has been completely performed [9] in the strong mass limit so that

$$
\begin{aligned}
Z_{T h}= & \int D A^{\mu} \exp \left[-\frac{1}{2} \int d^{3} x\left(\frac{1}{g^{2}}\left(A^{\mu}\right)^{2}+\frac{1}{4 \pi} A_{\mu} \epsilon^{\mu \nu \alpha} \partial_{\nu} A \alpha\right.\right. \\
& \left.\left.+\frac{1}{12 \pi m} F_{\mu \nu}^{2}+O\left((1 / m)^{2}\right)\right)\right] .
\end{aligned}
$$

The higher-order terms in the $1 / m$ expansion that lead to higher-derivative terms do not concerns us here.

Considering the zeroth-order term, the massive Thirring model in 3D is reduced to the self-dual model Lagrangian:

$$
S_{S D}=\int d^{3} x\left(\frac{1}{2 g^{2}} A^{\mu} A_{\mu}-\frac{1}{2} \epsilon^{\mu \nu \alpha} A_{\mu} \partial_{\nu} A_{\alpha}\right) .
$$

We see thus that the mapping between the Schwinger model and the dimensionally reduced 3D self-dual model points to a relation between the $3 \mathrm{D}$ Thirring and the $2 \mathrm{D}$ Schwinger models.

We consider once again, to clarify the reduction method, the dimensional reduction of this model (17) with an alternative procedure. We proceed now by rewriting the $A^{\mu}$ field in terms of its dual:

$$
A^{\mu}=\epsilon^{\mu \nu \alpha} \partial_{\nu} \tilde{A}_{\alpha} .
$$

Setting $\varphi=\widetilde{A}_{2}$, and assuming no dependence in $x^{2}$, the action (17) is reduced to a modified version of the bosonized Schwinger model:

$S=\int d^{2} x\left(-\frac{1}{4 g^{2}} \widetilde{F}_{i j} \tilde{F}^{i j}+\frac{1}{2 g^{2}}\left(\partial_{i} \varphi\right)^{2}+\frac{1}{2} \square \varphi \epsilon^{i j} \tilde{F}_{i j}\right)$.

The introduction of the first-order term in $1 / m$ as in Eq. (16) yields the additional terms

$$
S_{1}=\frac{1}{6 \pi m} \int d^{2} x\left(\frac{1}{2} \partial_{i} \varphi \square \partial^{i} \varphi-\frac{1}{4} \widetilde{F}_{i j} \widetilde{F}^{i j}\right)
$$

which correct the modified Schwinger action. The Lagrangian (19) coincides with the one that we have obtained by means of the dual interpolating mechanism for the Maxwell term in the Maxwell-Chern-Simons action and the direct dimensional reduction of the dual field (13) for the special value of the parameter $\gamma^{2}=\square$.

It is interesting to relate the modified Schwinger model (19) to a fermionic model in two dimensions. It is not difficult to guess that the fermionization of it will lead to the action of a canonical fermion with derivative coupling

$$
S_{f}=\int d^{2} x\left[\bar{\psi}(i / \partial-i e \square \tilde{\mathbb{A}}) \psi-\frac{1}{4}\left(\tilde{F}_{i j}\right)^{2}\right] .
$$

In order to see this, let us decouple the fermion field. We perform the chiral rotation

$$
\begin{aligned}
& \psi^{\prime}=e^{\gamma_{5} \square \phi+i \square \eta} \psi, \\
& \bar{\psi}^{\prime}=\bar{\psi} e^{\gamma_{5} \square \phi-i \square \eta},
\end{aligned}
$$

where $\widetilde{A}_{i}=\epsilon_{i j} \partial_{j} \phi+\partial_{i} \eta$. The Jacobian associated with this decoupling transformation can be computed with the Fujikawa method [10]. The Wick rotated $\left(x^{0} \rightarrow x^{2}\right)$ expressions of the Dirac operator and its adjoint are 


$$
\not D_{E}=\gamma^{2}\left(\partial_{2}+i e \square \tilde{A}_{2}\right)+\gamma^{1}\left(\partial_{1}+i e \square \tilde{A}_{1}\right)
$$

and

$$
D_{E}^{\dagger}=-\gamma^{2}\left(\partial_{2}+i e \square \tilde{A}_{2}\right)+\gamma^{1}\left(\partial_{1}+i e \square \tilde{A}_{1}\right) .
$$

By choosing the basis of eigenfunctions,

$$
\not D_{E} \not D_{E}^{\dagger} \xi_{n}=\lambda_{n}^{2} \xi_{n}
$$

the Jacobian of the transformation turns out to be expressible as

$$
J(x)=\exp \left(-2 i e \int d^{2} x \square \phi I(x)\right),
$$

with

$$
I(x)=\lim _{M \rightarrow \infty} \sum_{n} \xi_{n}^{\dagger} \gamma_{5} \xi_{n} e^{-\left(\lambda_{n}^{2} / M^{2}\right)} .
$$

A straightforward computation leads to

$$
I(x)=\frac{e}{4 \pi} \square \epsilon_{i j} \widetilde{F}_{i j},
$$

so that

$$
J(x)=\exp \left(-\frac{i e^{2}}{2 \pi} \int d^{2} x \widetilde{F}_{i j} \square \tilde{F}_{i j}\right) .
$$

The effective action for the vector field after integrating out the fermionic variable and returning to Minkowski spacetime turns out to be

$$
S_{\mathrm{eff}}=\int d^{2} x\left[-\frac{1}{4}\left(\tilde{F}^{i j}\right)^{2}-\frac{e^{2}}{2 \pi} \tilde{F}_{i j} \square \tilde{F} i j\right] .
$$

This effective action is the same as the one obtained from integration on the scalar field in the action (19), thus establishing the equivalence with the fermionic model (21).

We have obtained a series of relationships that occur among two-dimensional models having their origin in their relation to three-dimensional models. The topologically massive Maxwell-Chern-Simons action leads through dimensional reduction to the bosonized Schwinger model. The massive aspect of both models is obtained with basically the same mechanism. On the other hand, the Maxwell-ChernSimons action is related to the self-dual action through an interpolating field machinery. An extension of this machinery allows for its representation also in terms of a family of models which are in general nonlocal. For a specific member of this family instead of nonlocallity we obtain a model with higher derivatives. This relationship can also be understood as a relation among the two-dimensional model and the hypermassive Thirring model in three dimensions.

It would be interesting to try to develop a better understanding of the two-dimensional models that result from the dimensional reductions of the non-Abelian counterparts of the models here dealt with. In particular the non-Abelian Maxwell-Chern-Simons model will be led to the twodimensional Yang-Mills field interacting with the (pseudo)scalar field in the adjoint representation with the additional $e \epsilon^{i j} \phi F_{i j}$ gauge-invariant topological interaction term. Is there an interpretation of this model as the bosonized form of some $2 \mathrm{D}$ model? In addition, the bosonization in three dimensions raises the question of whether the dimensional reduction of the expression of the bosonized fermion field in three dimensions [11,5] is related to the Mandelstam formula. Another interesting aspect of dimensional reduction is whether useful information about the charge and flux carried by particles in 3D models may be obtained starting from their corresponding 2D models. These questions are presently under investigation.
[1] S. Deser, R. Jackiw, and S. Templeton, Phys. Rev. Lett. 48, 975 (1982); Ann. Phys. (N.Y.) 140, 372 (1982).

[2] A. N. Redlich, Phys. Rev. D 29, 2366 (1984).

[3] J. Schwinger, Phys. Rev. 128, 2425 (1962); J. H. Lowenstein and J. A. Swieca, Ann. Phys. (N.Y.) 68, 112 (1971).

[4] E. Fradkin and F. A. Shaposnik, Phys. Lett. B 338, 253 (1994); S. Deser and R. Jackiw, Phys. Lett. 139B, 371 (1984).

[5] P. R. Townsend, K. Pilch, and P. V. Nilwenhuizen, Phys. Lett. 136B, 32 (1984); C. P. Burgess, C. A. Lütken, and F. Quevedo, Phys. Lett. B 336, 18 (1994); K. Ilkegami, K. Kondo, and A. Nakamura, Prog. Theor. Phys. 95, 203 (1995); R. Banerjee, Phys. Lett. B 358, 297 (1995); Nucl. Phys. B465, 157 (1996).

[6] E. Fradkin, Field Theories of Condensed Matter Systems
(Addison-Wesley, Reading, MA, 1991).

[7] R. E. Gamboa-Saravi, F. A. Shaposnik, and J. E. Solomin, Nucl. Phys. B185, 239 (1981).

[8] R. Banerjee, H. J. Rothe, and K. D. Rothe, Phys. Rev. D 52, 3750 (1995).

[9] A. S. Dutra and C. P. Natividade, "Remarks on Fermion Boson equivalence in three dimensions," IF/UFF Report No. 010/98 th001/98.

[10] K. Fujikawa, Phys. Rev. Lett. 42, 1195 (1979); Phys. Rev. D 21, 2848 (1980).

[11] E. C. Marino, Phys. Lett. B 263, 63 (1991); D. Barci, C. D. Fosco, and L. E. Oxman, ibid. 375, 267 (1996); R. Banerjee and E. C. Marino, Nucl. Phys. B507, 501 (1997). 